\title{
DEVELOPING A MOBILE SERVICE-BASED CUSTOMER RELATIONSHIP MANAGEMENT SYSTEM USING FUZZY LOGIC*
}

\author{
Xiaobei LIANG ${ }^{1}$, Jianghua ZHANG $^{2, \dagger}$, Bingyong TANG $^{3}$ \\ ${ }^{1}$ School of Economics \& Management, Tongji University, 200092 Shanghai, P.R. China \\ ${ }_{2}^{2}$ School of Management, Shandong University, 250100 Jinan, P.R.China \\ ${ }^{3}$ Glorious Sun School of Business and Management, DongHua University, 200051 Shanghai, P.R.China \\ E-mail: liangxiaobeibei@gmail.com,zhangjianghua28@gmail.com,tangby@dhu.edu.cn
}

Received: 29-04-2010, Accepted: 05-10-2010

\begin{abstract}
Customer relationship management (CRM) has gained lately widespread popularity in many industries. With the development of economy and society, customers are unsatisfied with the stereotyped products. As customers usually describe their demands in nature language, the demands are often conflicting with each other and are often imprecise. The paper studies the operation process of handling customer demand for modern service systems based fuzzy logic methods. While in this mobile medium times, mobile service and CRM are rarely taken into unite study. This paper overviews the related theory likes business engineering, relationship marketing and mobile business, which can be used in mobile CRM (mCRM) and in the implement of mobile CRM. The paper analyzes the underlying technology and marketing issues of the initiation of mCRM and integrat es various issues of mCRM. Moreover, the paper discusses the characteristics of useful mCRM as the implement of mCRM will help the enterprise enhance the customer relationship and customers' loyalty will also gain more profit. A new stability criterion for the extended singular dynamic input-output model is given to ensure the stability of input-output model.
\end{abstract}

Keywords: customer demands, fuzzy logic, mobile customer relationship management (mCRM), mobile service, stability.

\section{Introduction}

Customer relationship management (CRM) has gained lately widespread popularity in many industries. Relying on the proposition that customer relationships are a true source of competitive advantage, the objective of CRM is to build and maintain customer relationships by encompassing the sales, marketing, and customer service activities.

The findings in Kalakota and Robinson show developing a new customer costs more than six times the effort of keeping a present one ${ }^{1}$. Hence, any demand of the existing customer needs to be treated more positively, mining changes in customer behavior for the modern service systems are very important ${ }^{2,3,4}$. While for most situations, due to insufficient technical knowledge and lack of awareness about specifications, customers may present demands with some inexact meanings. Many service managers revealed that the customer demands often received with verbally vague description and hard to handle. Thus, the fuzzy demands of the customers will lead to great difficulty in product design, for which two important issues need to be addressed. First, the existing customer demands the features of the current products. Second, customers' expectations are new product features. However, the voice of the cust omer is generally expressed in layman's language and not explicitly in terms of product features.

\footnotetext{
* This work is supported by grant 70832005 of the key Program of National Natural Science Foundation of China.

${ }^{\dagger}$ Corresponding author. School of Management, Shandong University, 250100 Jinan, P.R.China (Jianghua ZHANG).

Tel.: 0086-531-88363169, Fax: 0086-531-88364335. E-mail: zhangjianghua28@ gmail.com.
} 
To process such expressions, a method is needed to decompose and classify these expressions to enable the new demands to be explored.

To build and maintain long-term relationships with customers, companies should provide differentiated relationship values and communicate continuously and consistently with each of their customers. For this purpose, new digital marketing channels, such as the Internet and mobile phones, are considered powerful channels to reach customers because they allow personalization and interactivity of the content and the context of the messages ${ }^{5,6}$. Moreover, digital channels are seen to create unique and positive experiences to customers by mixing aspects of products, service, brand, and communication 7 , and making it possible for companies to develop interactive and relationshipbuilding contacts with their potential and current customers ${ }^{8}$. Despite the potential of digital channels, only the Internet as a channel to manage customer relationships has attracted a lot of attention among academics $^{9,10}$.

Now mobile services are more and more important for firms. However, firms can not invest all of resources in developing mobile services. In fact, customs use mobile devices mainly for the simplest services, such as text messaging or voice services. Then, understanding the amount putting in mobile services and the quantity of output is very necessary. To prevent the spread of economic crisis, economists want ed to study the current state of a company and research how various policies can be used to move the system from its present status to a future more desirable state when they tried to deal with those dynamic systems. Furthermore, real economic systems have uncertain and unpredictable factors. These factors must be considered when inputoutput problems are researched. In this paper, we will directly deal with a kind of extended singular inputoutput system on mobile services instead of convert ing them into general systems and consider the uncertainties existing in real companies.

\section{Literature Review}

\subsection{Semantic analysis and evaluation model}

The customer demands are normally expressed using natural language mostly in layman's terms. For example, customers may require " easy to handle" or "good performance" for a new car. When collecting information on product features from a large group of customers, computerization of the data processing is needed to improve the understanding of customer demands and reduce the processing time. However, the use of computers can result in difficulties in directly extracting information from the customers' expressions. Such difficulties have been frequently discussed, and many applications or techniques have been developed to solve this problem. Among them, Natural Language Processing (NLP) provides a feasible solution ${ }^{11}$. In the field of Artificial Intelligence (AI), the focus of NLP is on the knowledge necessary to understand natural language. AI deals with language as aphenomenon of knowledge representation and use. The objective of NLP can be achieved by using syntactically driven parsing and semantic grammar ${ }^{12}$.

Evaluation is a process that analyzes elements in order to achieve different objectives such as quality inspection, marketing and other fields in industrial companies $^{13}$. In these evaluation processes the information provided by the experts implies uncertainty, vagueness and imprecision. The use of the Fuzzy Linguistic Approach has provided successful results modeling such a type of information. In sensory evaluation it may happen that the panel of experts have more or less degree knowledge of about the evaluated items or indicators. So, it seems suitable that each expert could express their preferences in different linguistic term sets based on their own knowledge $e^{13}$.

Success of an e-business company is strongly associated with the relative quality of its website compared to that of its competitors. The purpose of this study is to propose a multi-attribute e-business website quality evaluation methodology based on a modified fuzzy TOPSIS approach. In the proposed methodology, weights of the evaluation criteria are generated by a fuzzy AHP procedure. In performance evaluation problems, the judgments of the experts may usually be vague in form ${ }^{14}$.

\subsection{Ranking or prioritizing customer demands}

Two methods being used most to ranking the customer demands: potential gain in customer value index, analytic hierarchy process.

Hom proposed the Potential Gain in Customer Value (PGCV) index, which is an extension of a common marketing analysis method ${ }^{15}$. In such an analysis, a survey is designed to obtain customer ratings on the 
importance of certain product features and their performance. Through the survey, the priority order of product features is measured based on two essential dimensions: (i) the customers' perception of the importance of the product features; and (ii) the performances of the individual features. The two dimensions form an Importance/Performance (IP) chart. The location in the four quadrants (A, B, C and D) of an IP chart denotes the strategic implication of the product features.

The customer requirements are decomposed into a hierarchical structure. The relative importance of customer requirements at each level is determined by a pair-wise comparison. The relative importance of each requirement at the lower level is multiplied by the priority weight of its upper level. The final priorities of the customer requirements are calculated using this bottom-up procedure.

Classification based on association rules is considered to be effective and advantageous in many cases. However, there is a so-called "sharp boundary" problem in association rules mining with quantitative attribute domains. Aiming at proposing an associative classification approach, namely Classification with Fuzzy Association Rules (CFAR), where fuzzy logic is used in partitioning the domains. In doing so, the notions of support and confidence are extended, along with the notion of compact set in dealing with rule redundancy and conflict ${ }^{16}$.

The decision making problems may include preference information in different formats. A latticebased linguistic-valued weighted aggregation (LVWA) operator is proposed for multiple attribute group decision making with non-totally ordered linguisticvalued information. Then some transformation functions for unifying different formats of preference information are reviewed and summarized ${ }^{17}$.

\subsection{Relationship marketing}

Several authors have clearly pointed towards a high level of association between CRM and relationship marketing ${ }^{18,19,20}$, and some authors do not even make any distinction between the terms ${ }^{21,22}$. This emphasis on relationships is redefining how companies are interacting with their customers ${ }^{23,24}$. On the other hand, elements common to all definitions of CRM include leveraging technology to engage individual customers in a meaningful dialogue so that firms can customize their products and services to attract new customers, develop relationships, and retain existing customers ${ }^{25}$. While CRM does not acknowledge the technology underlying the management of customers, CRM is described as using information technology (IT) in implementing relationship marketing strategies ${ }^{26}$. As such, CRM unites the potential new technologies and RM thinking to deliver profitable, long term relationships ${ }^{27}$.

\subsection{Mobile business}

Technological advancements in mobile communications enable new ways of doing business ${ }^{28}$, often referred to as "mobile business" (MB) or "mobile commerce" (MC). While Turowski/Pousttchi ${ }^{29}$ do not distinguish between the two but rather use the term "mobile commerce", Lehner ${ }^{30}$ defined " mobile business" as the application of mobile technologies to improve or extend business processes and open new market segments. They differentiated between "mobile business" and "mobile commerce", the latter being a rather subordinate MB field focusing on the handling of transactions. We follow Lehner and Zobel's more general understanding and concentrate on mobile technologies' application to support CRM processes (mCRM). Typical examples of mCRM are mobile marketing (MM), mobile sales force automation (MSA) and mobile field service (MFS), or mobile customer service (MCS). MM takes advantage of mobile devices' pervasiveness and the very personal nature that is typical of communication when using those devices ${ }^{31}$. MSA and MFS support sales agents and field service agents whose work is generally spread across multiple locations by giving them access to CRM systems and other required information from any location. MCS usually gives customers ubiquitous access to services to which they have subscribed ${ }^{32,33}$.

\section{Processing the Customer Demand}

There are four characters of customer demands:

- Fuzzy. The demands are usually fuzzy and indeterminate. Customers' demands about the production are ambiguous and unspecific. Customers often use words like "less than", "in some sort" to express their demands.

- Dynamic. The demands change all the time. On the one side, the customer demands will impenetrate the whole life cycle, which shows different forms in the different phases. On the other side, most 
customers change their demands with the different entironment.

- Multiformity. The multiformity of customer demands lay on two sides: (1) from the overlay point of view. The customer demands include demands in product designing, manufacturing management and performance etc. (2) from the expressing method. The customer demands express not only in natural language, sometimes also use figure, table and symbol to express the demands.

- Priority. The demands ranking different among each other. The importance and satisfaction of customer demands are different. Some basic function demands are extremely important and some technology parameters are slightly important. According to the characteristics of the customer demands, we use the following method to processing the demands.

\subsection{Demand unit analysis tree}

As we discussed above, the customer demand are fuzzy, inexact and some times antinomy. We should decompose the demands into sub-demands. The decomposition of demands will help to set the demands model, define the customer demands information and made it easy to understand. Here, we defined demand unit as the smallest information unit which is indivisibility and can describe the customer demand unambiguously ${ }^{34}$.

Customer demands have hierarchy. The customer demands model can be constructed by decomposing the demands according to hierarchy relations ${ }^{35}$. In this paper, we use analysis tree to express the connections between demands hierarchy structure. We define the demand root, demand unit and sub-demand as follows (see Fig.1):

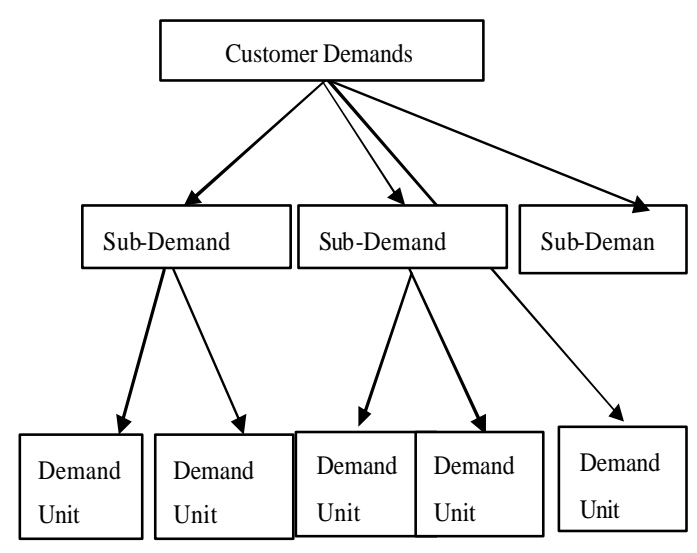

Fig.1. Demand unit analysis tree

\subsection{Demand decomposition}

We should decompose the original fuzzy and unambiguous customer demand to model the analysis tree. There are two important questions: How to control the decompose dimension, when should we stop decomposition. How to decompose demands?

The process of demand decomposition can be figured as (see Fig.2) :

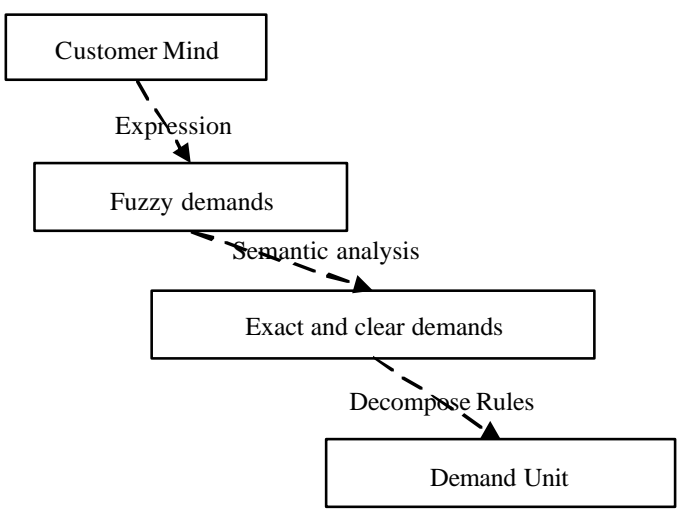

Fig.2. Process of Demand Decomposition

If $d_{1}, d_{2} \ldots d_{n}$ are the demand units, $\Phi$ means there are no repetition between the demand units. The rules of decomposition can be expressed as:

$$
\begin{aligned}
& D E(d) \subset D E\left(d_{1}\right) \wedge D E\left(d_{2}\right) \wedge \ldots \wedge D E\left(d_{n}\right) \\
& \left(\forall d_{i}, d_{j}\right) D E\left(d_{i}\right) \wedge D E\left(d_{j}\right)=\Phi(i \neq j)
\end{aligned}
$$

Customers usually use natural language to describe their expectation. These describe language includes the customers' demands for the products. We can mine the 
demand in the words through semantic rules. For example, the customer demands for clothes (see Fig.3):

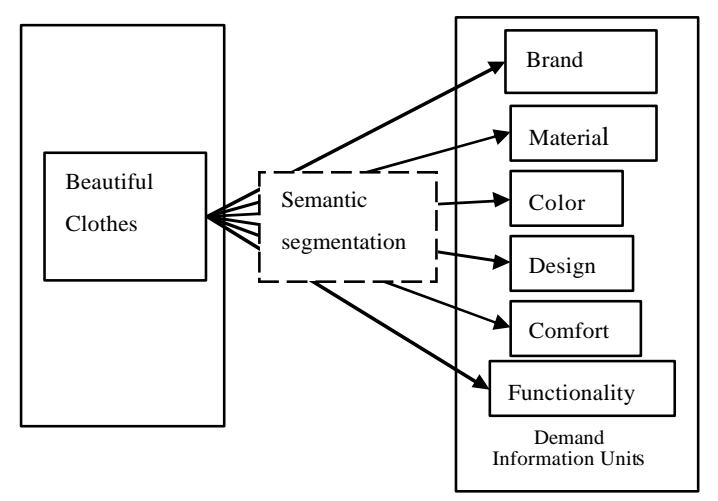

Fig.3. Demand semantic segmentation

\subsection{Customer demand priority rating}

To determine the priority of demand units, the fuzzy IFThen rules should be customer driven. The action part of the rules should be able to reflect customer needs for a specific demand unit. The demand unit with high importance ratings should have higher priorities than those with low importance rating. The priority should be discussing together with the customer satisfaction. We can represent the relationship between importance, satisfaction and priority:

Table 1. The relationship between importance, satisfaction and priority.

\begin{tabular}{|c|c|c|c|}
\hline \multirow{2}{*}{ Importance } & \multicolumn{3}{|c|}{ Satisfaction } \\
\cline { 2 - 4 } & Low & Medium & High \\
\hline High & $\begin{array}{c}\text { Extremely } \\
\text { high }\end{array}$ & Very high & High \\
\hline Medium & $\begin{array}{c}\text { Slightly } \\
\text { high }\end{array}$ & Medium & $\begin{array}{c}\text { Slightly } \\
\text { low }\end{array}$ \\
\hline Low & Low & Very low & $\begin{array}{c}\text { Extremely } \\
\text { low }\end{array}$ \\
\hline
\end{tabular}

According to the table, the rules are developed:

(1) IF importance is high AND degree of satisfaction is low, THEN priority is extremely high.

(2) IF importance is high AND degree of satisfaction is medium, THEN priority is very high.

(3) IF importance is high AND degree of satisfaction is high, THEN priority is slightly high.
(4)IF importance is medium AND degree of satisfaction is low, THEN priority is high.

(5) IF importance is medium AND degree of satisfaction is medium, THEN prority is medium.

(6) IF importance is medium AND degree of satisfaction is high, THEN priority is slightly low.

(7) IF importance is low AND degree of satisfaction is low, THEN priority is low.

(8) IF importance is low AND degree of satisfaction is medium, THEN priority is very low.

(9) IF importance is low AND degree of satisfaction is high, THEN priority is extremely low.

\section{The Implement of MCRM}

\subsection{Technology issues of mCRM}

Technology plays the substantial role in CRM. Because this kind of information system development often includes major uncertainties, the technological issues have an even more vital role in the initiation of mCRM. In addition, customers will not begin using their mobile phones for new functions simply because new technology exists. Despite the key challenge is to get the customers to open and use the company's mobile portal. To sum up, to start the mCRM companies have to build the technology underlying mCRM and, accordingly, solve the technological uncertainties related to it. There are four critical issues to discuss (see Fig.4).

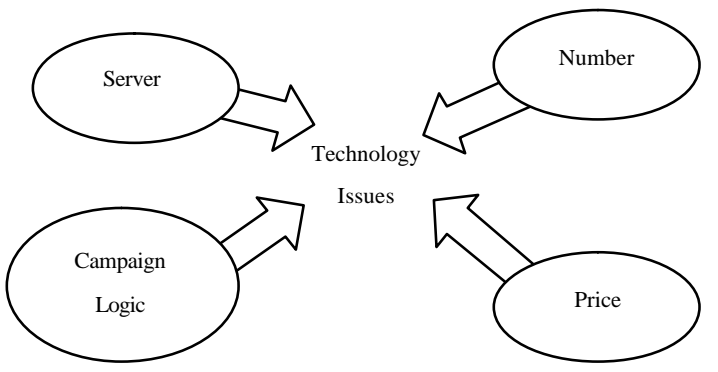

Fig.4. Technology Issues of mCRM

- Server. The company had to acquire mCRM server to be capable of handle, send, receive and store amount of SMS and MMS messages. Company has to decide either to build in-house expertise or rely on partners, and whether to use a managed or a hosted solution. In this case, the company decided to start mCRM with a hosted mobile marketing 
platform provided by one of leading companies in the mobile marketing and mCRM applications market.

- Number. The company had to decide how to acquire a short message service number which directs SMS messages from mobile phones to mCRM server. Basically, the target audience for every campaign will span all major networks of a particular country. Consequently, the campaign must connect to all networks.

- Campaign Logic. Campaign logic refers the details that customers are supposed to answer the details that asked from the customers during the campaign. Without the campaign logic, he server cannot receive and store messages sent by the service users. In addition, the data received from customers is almost impossible to turn into customer information without the logic. To be sure that relevant information will be saved to the database, an accurate plan will have to be made.

- Price. B asically there are three options for setting up the price per message sent by service user: normal SMS price, free SMS and premium-rate SMS message. The company should decide which service to use.

\subsection{Marketing issues of $\mathrm{mCRM}$}

From technically implemented point of view, the key challenge is to find marketing means to attract customers' attention and gain them to initiate dialogue with the company in question. At this marketing level, there are two issues todiscuss as in Figure 5.

- Customers' attention. How to attract the attention of the potential customers to use mobile medium in communication. Because the attraction is pursued by the aid of the other mediums, the initiation of mCRM for the very first time has, therefore, the same basic principles as any other traditional marketing campaign.

The company must identify the target audience, determine communication objectives, design the message, choose the medium through which to lure customers' attention, and finally collect feedback to measure the promotion's results. When considering mCRM after the initiation, a concept should be incorporated into the overall marketing strategy. To undertake a new series of campaigns based merely on the mobile medium or integration mobile medium to existing marketing channels, the latter option, where the mobile medium is integrated to existing marketing campaigns on other media has proven to be the most effective and efficient. In other words, mCRM campaigns complement other media, such as television, print and Internet, and vice versa. In this case, the newspaper was used to attract the customer to join into the mCRM campaign.

- Data processing. Where they obtained the details of the target group from and whether they are in fact able to send mCRM messages to these people because of legal constraints. Basically, there are three different ways to obtain a database of opt-in mobile numbers; purchasing, renting or developing the database of your own. The company in question decided to develop or collect the database independently, in order to get prior permission to start mobile dialogue with its customers.

The collection of the database was organized around a marketing campaign, where the company was asking customers to send their personal information, such as mobile phone numbers, as well as names and addresses, preferences and a permission to send messages to end user's mobile phone via SMS to the retailer. The customers could also sign up to the loyalty program. By signing in for the loyalty program, customers automatically opt-in to the company's permission based mCRM database. The campaign was successful as almost 18000 out of over 22000 respondents (approximately over 80 percent) signed up to the loyalty program and gave their permission. The incentive for the customers to give their personal information was a chance to win a brand new car in a lottery. In addition, valuable information and special offers promised to send via SMSthose, who gave permission.

\subsection{Integrating issues}

In addition to these technology and marketing related issues discussed above, mCRM has to be integrated with existing CRM system, as the MCRM solution chosen must have ability to provide complete integration with the existing CRM system. This system integration is necessary condition for achieving effective management of interactions across and information coordination between different customer touch points (e.g., Internet, direct mail, sales call, and mobile medium). Naturally, the integration is necessary prerequisite for mCRM upon the existing CRM database. Nowadays, extending CRM to mobile medium is not as complicated and many CRM systems already 
support the extension of CRM to mobile medium (see Fig.5).

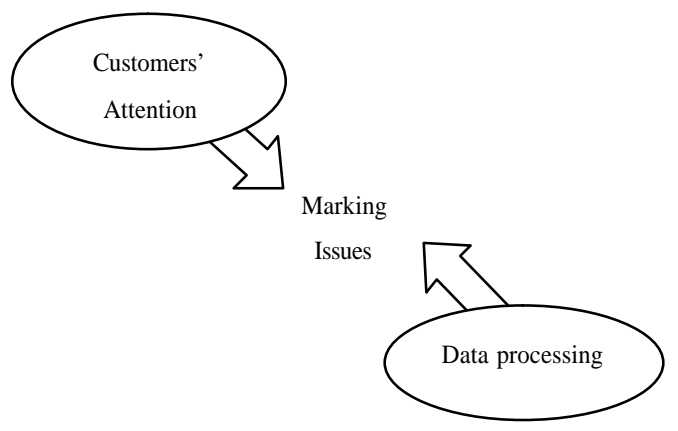

Fig. 5. Marketing Issues of mCRM

\subsection{Characteristics for successful mCRM}

The success of mCRM is not only depending on technological maturity of mobile technology and the penetration of mobile devices. The predictions are hard to be made, but there are certain factors that have to be considered when developing a mobile service. Mobile CRM should provide added value arising from the mobility of the new medium. Key drivers for mCRM today are:

- Ubiquity---communication anywhere, independent of the user's location.

- Reachability---users can be contacted anywhere anytime or they can limit their reachability to particular persons or times.

- Security-mobile security technology is emerging in terminal providing identification of the owner.

- Convenience---devices store data, are always at hand and are increasingly easy to use.

- Enhanced functionality which is already partially available must support the following factors:

- Localization of services and applications

- Instant Internet Connectivity from a mobile phone

- Personalization

The value of mobile CRM in business sector is studied extensively, since this significance of mobile business applications is predicted to increase in the near future. Eight core benefits of the mobile business applications are summarized in: Connectivity, Flexibility, Ubiquity, Location Awareness, Interactivity, Efficiency, Effectiveness andBusiness Transformation.

\section{Stability of Input-output Model for Mobile Services}

\subsection{Economics model}

In this section, the classic dynamic Leontief inputoutput model will be extended to a kind of singular and stochastic input-output model to describe the inputoutput amount of firms on mobile services.

Consider the classic Leontief input-output model described by

$x(k)=A x(k)+B[x(k+1)-x(k)]+Y(k)$.

The vector $x(k)=\left[\begin{array}{lllll}x_{1}(k) & \mathrm{L} & x_{i}(k) & \mathrm{L} & x_{n}(k)\end{array}\right]^{T}$ is the total output vector and $x_{i}(k)$ is the total output from sector $i . Y(k)$ is the final net product vector. The matrix $A$ is the direct consumption coefficient matrix, $B$ is the capital coefficient matrix. This model is discret etime input-output system. The discrete-time inputoutput models are used widely because nations make the economic plans one time every year or every month. However, some economic actions, such as the production of a factory, are often continuous. So the continuous-time dynamic input-output model should be investigated. If we replace the difference $[x(k+1)-x(k)]$ with the differential $x \&(t)$, the classic Leontief input-output system (1) will be turned into continuous-time dynamic Leontief input-output model:

$x(t)=A x(t)+B \&(t)+Y(t)$

The vector $Y(t)$ can be considered as the Leontief input-output model's control vector because we can affect the quantity of final net product by many economic management methods such as controlling the scale of investment. Then $x(t)$ can be treated as the state vector. Thus the dynamic Leontief input-output model (2) can be turned into a state space model. In real companies on mobile services, capital coefficient matrix $B$ is not always invertible, because the products of some sectors of companies, such as after-sale service departments, can not be treat as capital product and applied to invest. So the dynamic Leontief input-output model (2) can be rewritten as

$B \&(t)=(I-A) x(t)-Y(t)$

where $\operatorname{rankB}=r<n$.

In real economic systems, there are many unpredicted factors. When the disturbing factors are considered, the traditional input-output model should be extended to describe the real economic systems. The 
parameter uncertainty is introduced and the parameters are considered as the Markovian jumping parameters. In other words, the coefficient matrices $A$ and $B$ will be considered to Markovian jumping parameters. Furthermore, the investment to a company can not have the effect at once. So the time lag need be considered. Then the system(3) turns into

$$
B(\mathrm{r}(\mathrm{t}))(t)=(I-A(\mathrm{r}(\mathrm{t}))) x(t)+A d(r(t)) x(t-1)-Y(t)
$$

The vector function $r(t)$ is a random function representing a continuous-time discrete-state Markovian process which takes value in a finite set $M=\{1,2,3 \mathrm{~L}, s\}$. In economic society, one important problem is about the quantity of final net product. Sometimes, the final net product is hoped to increase to a higher level several years later. However, the quantity of final net product should not increase forever because resources of the earth are scarce. Scarcity means that society has limited resources and therefore can not put all of resources to one sector. So it is hoped that the quantity of the final net product remains in a quite stable level. So, we need research the stability of the extended uncertain Leontief input-output model (4) on mobile services-system .

Where, $\mathbf{R}^{n}$ is the $\mathrm{n}$-dimensional Euclidean space; $\mathbf{R}^{m \times n}$ denotes the $m \times n$ real matrices space; $I$ is the $n \times n$ identity matrix; $A^{T}$ denotes the matrix transposition; "* * is used as the term that are introduced by symmetry; when matrices $X$ and $Y$ are symmetric, $X>Y$ means that matrix $(Y-X)$ is positive-definite; $\Omega$ is a compact set.

\subsection{Stability of inputoutput model}

This section will investigate the stability of the system (4). To discuss this problem, the following definition is necessary.

Definition 1: The extended Leontief input-output model is described by equation (4). $r(t)$ can be rewritten as $i=r(k)$.

1) System (4) is called to be regular

if $\operatorname{det}\left(s B_{i}-I+A_{i}\right)$ and $\operatorname{det}\left(s B_{i}-I+A_{i}-A d_{i}\right)$ is not

identically zero for any $i$.

2) System (4) is impulse-free

if $\operatorname{deg}\left(\operatorname{det}\left(s B_{i}-I+A_{i}\right)\right)=\operatorname{rank} B_{i}$ and

$\operatorname{deg}\left(\operatorname{det}\left(s B_{i}-I+A_{i}-A d_{i}\right)\right)=\operatorname{rank} B_{i}$.

3) System (4) is asymptotically stable if

equation $B_{i} \&(t)=\left(I-A_{i}\right) x(t)+A d x(t-1)$ is

asymptotically stable for any $i$.
4) System (4) is called to be asymptotically admissible if it is regular, causal and asymptotically stable.

Then we have the following result.

Theorem 1: System (4) is asymptotically admissible if there exist matrices $\mathrm{P}_{\mathrm{i}}>0, \mathrm{Q}_{\mathrm{i}}>0, \mathrm{Z}>0, \mathrm{X}_{11 \mathrm{i}}=0, \mathrm{X}_{12 \mathrm{i}}=0$, $X_{2 i_{i}}=0, N_{1 i}, N_{2 i}(i=1,2, \cdots, s)$, such that the following inequality holds:

$$
\begin{aligned}
B_{i}^{T} P_{i} & =P_{i}^{T} B_{i} \geq 0 \\
\Phi & =\left[\begin{array}{ccc}
\phi_{11} & \phi_{12} & Z-A_{i}^{T} Z \\
* & \phi_{22} & A d_{i}^{T} Z \\
* & * & -Z
\end{array}\right]<0 \\
\Psi & =\left[\begin{array}{ccc}
X_{11 i} & X_{12 i} & N_{1 i} \\
* & X_{22 i} & N_{2 i} \\
* & * & Z
\end{array}\right] \geq 0 \\
\text { where } & \\
\phi_{11}= & 2 P_{i}-P_{i} A_{i}+A_{i}^{T} P_{i}+Q_{i}+N_{1 i} B_{i}+B_{i}^{T} N_{1 i}^{T}+X_{11 i}+\sum_{j=1}^{s} \pi_{i j} B_{i}^{T} P_{j} \\
\phi_{12}= & P_{i} A d_{i}-N_{1 i} B_{i}+B_{i}^{T} N_{1 i}^{T}+X_{12 i} \\
\phi_{22}= & -N_{1 i} B_{i}-B_{i}{ }^{T} N_{1 i}^{T}+X_{22 i}-Q_{i} \cdot
\end{aligned}
$$

The proof can be shew in reference 36 .

\subsection{Numerical example}

For the purpose of simulating the run of the discrete singular input-output model, a numerical example is provided. Consider the dynamical input-output model (1), parameters are assumed as followed:

$$
B=\left[\begin{array}{cc}
0.5 & 0.6 \\
0 & 0
\end{array}\right], A=\left[\begin{array}{cc}
-0.3 & -1.4 \\
3.45 & 4
\end{array}\right]
$$

When $\bar{Y}(k)=0$, in other words, all products are treat ed as capital product and applied to invest, the open-loop economic system (1) turns into

$$
\left[\begin{array}{cc}
0.5 & 0.6 \\
0 & 0
\end{array}\right] x(k+1)=\left[\begin{array}{cc}
1.8 & 2 \\
-3.45 & -3
\end{array}\right] x(k) \text {. }
$$

Obviously the open -loop system (8) is unstable (see Fig. 6). Our goal is the design of a state feedback. It can be verified that as follows:

$$
S=\left[\begin{array}{ll}
0 & 1
\end{array}\right]^{T} \quad Q=\left[\begin{array}{ll}
1 & 1
\end{array}\right]^{T}
$$




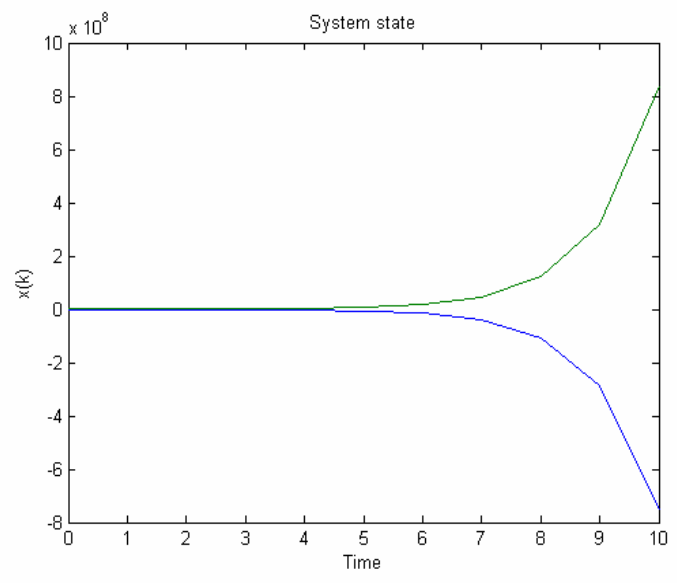

Fig. 6. State curve of open-loop system

$$
P=\left[\begin{array}{cc}
1.014 & 0 \\
0 & 1.0055
\end{array}\right], R=\left[\begin{array}{cc}
-4.7 & -4 \\
3 & 3
\end{array}\right]
$$

Therefore, a state feedback controller can be designed as follows:

$$
\bar{Y}(k)=\left[\begin{array}{cc}
-4.7 & -4 \\
3 & 3
\end{array}\right] x(k) .
$$

When applying this controller, the system (1) turns into

$$
\left[\begin{array}{cc}
0.5 & 0.6 \\
0 & 0
\end{array}\right] x(k+1)=\left[\begin{array}{cc}
6.5 & 6 \\
-6.45 & -6
\end{array}\right] x(k) \text {. }
$$

It is easily to show that the closed-loop discrete-time singular dynamic input-output system (9) is stable (see Fig. 7).

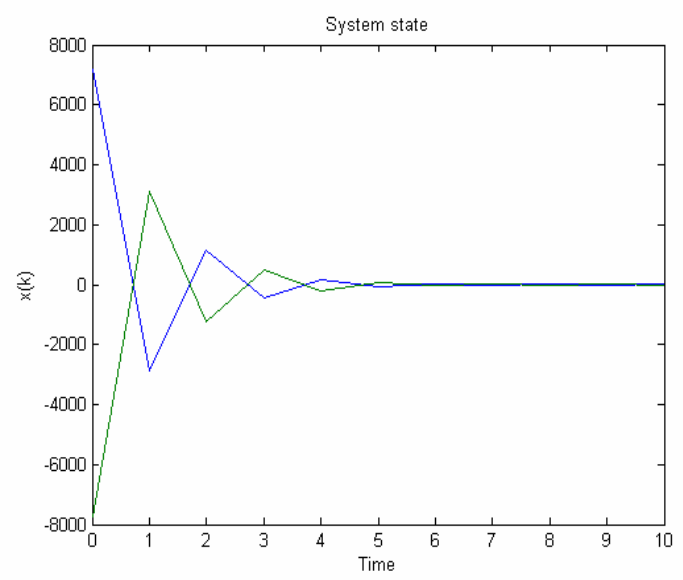

Fig. 7. State curve of closed loop system

\section{Conclusions}

This research mainly achieved in processing of customer demands for the modern service systems. Firstly, defined the demand unit and modeling the demand unit analysis tree. Secondly, use semantics to decompose the demands. Thirdly, we determined the priority of demand units. The result shows that although customer demands are usually described in layman's terms, they can still be processed by using semantics analyses. And given the ratings of importance and degree of satisfaction for cust omer demands, the fuzzy method can be used to determine their priority. Based on the priority of demands, the manufacturer can adjust their product design and reduce the risk of customer dissatisfaction.

In this paper we also discussed about mobile CRM.

We first mentioned some related theory such as business engineering, relationship marketing and mobile business. Based on the related theory, the paper discussed the main technology and marketing issues of mCRM and put forward the characteristics for a succe ssful mCRM. The future studies could develop the present conceptual mCRM and use it to help the company enhance the customer relationship, customers' loyalty, also gain more profit. A new stability criterion for the extended singular dynamic input -output model is given to ensure the stability of input-output model.

\section{References}

1. Kalakota, R., Robinson, M., E-business: Roadmap for Success, Addison-Wesley Publishing Co, New Jersey, 1999.

2. H. Chang, L. Hung and C. Ho, “ An anticipation model of potential customers' purchasing behavior based on clustering analysis and association rules analysis", Expert Systems with Applications, 32(3), 753-764(2007).

3. Afef Denguir-Rekik, Jacky Montmain, Gilles Mauris, “A possibilistic-valued multi-criteria decision-making support for marketing activities in e-commerce: Feedback Based Diagnosis System", European Journal of Operation Research, 195(3), 876-888(2009).

4. Huang Bingquan, Buckley B, Kechadi T.M, "Multi objective feature selection by using NSGA II for customer churn prediction in telecommunications", Expert Systems with Applications, 37(5), 36383646(2010).

5. Anders Henten, Hanne Westh Nicolajsen, “ Mobile and wireless communications: Technologies, applications, business models and diffusion", Telematics and Informatics, 26(3), 223-226(2009). 
6. Bouwman, H., De Vos, H., Haaker, T. (Eds.), Mobile Service Innovation and Business Models, Springer, Berlin, 2008.

7. Simova J, “Differentiated customer relationship management according to customer value in companies operating in the service sector", Economic and Management, 2, 118-127(2007).

8. Tseng TL, Huang CC, "Rough set-based approach to feature selection in customer relationship management", Omega, 35(4), 365-383(2007).

9. Chang Chewei, Chin-Tsai Lin, Wang Lian-Oing "Mining the text information to optimizing the customer relationship management", Expert Systems with Applications, 36(2), 1433-1443(2009).

10. Menger J., "CRM and Marketing: Customer orientation and relationship management information", Information Management and Consulting, 3, 37- 40(2007).

11. Obermeier, K.K, Natural Language Processing Technologies in Artificial Intelligence, Ellis Horwood, Chichester, West Sussex, England, 1989.

12. Carbonell, J.G., Natural Language Understanding, in Encyclopedia of Artificial Intelligence, Wiley, New York, 1992.

13. Martinez, Luis, Espinilla, Macarena, Perez, Luis G., "A linguistic multigranular sensory evaluation model for olive oil", International Journal of Computational Intelligence Systems 1 (2), 148-158(2008).

14. Kaya, Tolga, "Multi-attribute evaluation of website quality in e-business using an integrated fuzzy AHPTOPSIS methodology", International Journal of Computational Intelligence Systems, 3(3), 301-314(2010).

15. Armacost, R.L., Componation, P.J., Mullens, M.A. and Swart, W.W., "An AHP framework for prioritizing customer requirements in QFD: an industrialized housing application", IIE Transactions 26(4), 72-79(1994).

16. Chen Zuoliang, Chen Guoqing, "Building an associative classifier based on fuzzy association rules", International Journal of Computational Intelligence Systems 1(3), 262-273(2008).

17. Li, Xiaobing Ruan, Da Liu, Jun, Xu Yang, "A linguistic-valued weighted aggregation operator to multiple attribute group decision making with quantitative and qualitative information", International Journal of Computational Intelligence Systems 1(3), 274-284(2008).

18. Hom, W.C., " Relationship marketing and human capital”, Quality Progress, 30(3), 89-94(1997).

19. Ryals, L. and Knox, S., "Cross-functional issues in the implementation of relationship marketing through customer relationship management", European Management Journal, 19(5), 534-542(2001).

20. Christopher, M., Payne, A., and Ballantyne, D., Relationship Marketing: Creating Shareholder Value, Butterworth, Oxford 2002.

21. Jain, D. and Singh, S.S., "Customer lifetime value research in marketing: A review and future directions", Journal of Interactive Marketing, 16(2), 34-46(2002).
22. Chulhyun Kim, Suhwan Choe, Changwoo Choi, Yongtae Park, "A systematic approach to new mobile service creation", Expert Systems with Applications 35(3), 762 $771(2008)$

23. Gummesson, E., Total Relationship Marketing, Butterworth, Oxford, 1999.

24. Sheht, J.N. and Parvatiyar, A., The Handbook of Relationship Marketing, Thousand Oaks, Sage Publications, California, 2000.

25. Campbell, A.J., "Creating customer knowledge competence: managing customer relationship management programs strategically", Industrial Marketing Management, 32(5), 375-383(2003).

26. Ryals, L. and Payne, A., "Customer relationship management in financial services: towards informationenabled relationship marketing", Journal of Strategic Marketing, 9(1), 3- 27(2001).

27. Payne, A. and Frow, P., "The role of multichannel integration in customer relationship management", Industrial Marketing Management, 33(6), 527-538(2004).

28. Raisinghani, M., " Mobile commerce: transforming the vision into reality", Information Resources Management Journal, 15(2), 3-4(2002).

29. Turowski, K. and Pousttchi, K., Mobile Commerce: Grundlagen und Techniken, Springer, Berlin, 2003.

30. Lehner, F., Mobile und drahtlose Information system: Technologien, Anwendungen, Markte, Springer, Berlin, 2003.

31. Reichold, A., Schierholz, R., Kolbe, L. M. and Brenner, W., "M-Commerce at helsana health insurance: mobile premium calculator", Proc. of the DEXA '03 - Workshop Mobile Commerce Technologies and Applications, ed. Martin, D. C, 877-881(2003).

32. Looney, C. A., Jessup, L. M. and Valacich, J. S., "Emerging business models for mobile brokerage services", Communications of the ACM, 47(6), 7177(2004).

33. Mallat, N., Rossi, M. V. K., “ Mobile banking services", Communications of the ACM, 47(5), 23-35(2004).

34. D.Magro, P.Torasso, "Description and configuration of complex technical products in a virtual store", ECA2000 Workshop on Configuration, Berlin, 2000.

35. Barry O' Sullivan, "Con\$raint-based product structuring for configuration", Proc. of the 15th European Conference on Artificial Intelligence, IOS Press, Amsterdam, 41-46(2002).

36. Xiaohong Zhao, Fuyuan $\mathrm{Xu}$ and Bingyong Tang, "Stability of extended uncertain leontief input-output model on mobile services", Journal of Natural Science of Heilongjiang University, 27(4), 430434(2010). 